\title{
Complication and remission rates after endoscopic argon plasma coagulation in the treatment of haemorrhagic radiation proctitis
}

\author{
S. L. Siow ${ }^{1,2}$ • H. A. Mahendran ${ }^{1}$ - C. J. Seo ${ }^{1}$
}

Accepted: 8 August 2016

(C) Springer-Verlag Berlin Heidelberg 2016

\begin{abstract}
Purpose The management of haemorrhagic radiation proctitis is challenging because of the necessity for repeated intervention. The efficacy of argon plasma coagulation has been described before but the optimum treatment strategy remains debatable. This is a review of our experience over a decade treating patients with haemorrhagic radiation proctitis and their follow-up.

Methods This is a retrospective review of consecutive patients who underwent argon plasma coagulation for haemorrhagic radiation proctitis between January 2003 and December 2013. The patients were followed up using a prospectively maintained database.

Results Ninety-one patients were included with a mean follow-up of 13.1 months. Majoity $(n=85,93.4 \%)$ of the patients were female. Mean age at the time of treatment was 58.2 (range 23-87) years old. Majority of the patients ( $n=73$, $80.2 \%$ ) received radiotherapy for gynaecological malignancies followed by colorectal $(n=13,14.3 \%)$ and urological $(n=5,5.5 \%)$ malignancies. Mean interval between radiation and proctitis was 13.8 (range 3-40) months. Seventy-nine percent of patients were successfully treated after 1-2 sessions. Seventeen (18.7\%) patients experienced self-limiting early complications, and three (3.3\%) had late complications of rectal stenosis which was managed conservatively. Severity
\end{abstract}

S. L. Siow

szeli18@yahoo.com

1 Department of Surgery, Sarawak General Hospital, Kuching, Sarawak, Malaysia

2 Department of Surgery, Faculty of Medicine and Health Sciences, Universiti Malaysia Sarawak, Kota Samarahan, Kuching, Sarawak, Malaysia of bleeding during the initial presentation is an independent factor that predicts the number of sessions required for successful haemostasis $(p=0.002)$.

Conclusions Argon plasma coagulation is a reasonable treatment option in patients with haemorrhagic radiation proctitis with good safety profile. Our study suggests that the number of APC sessions required to arrest bleeding correlates with the severity of bleeding on initial presentation.

Keywords Haemorrhagic radiation proctitis · Argon plasma coagulation $\cdot$ Endoscopic treatment

\section{Introduction}

Radiation proctitis is a serious complication seen in patients who have received irradiation during the course of treatment of tumours located in the pelvis. It can be classified as either acute or chronic according to clinical presentation and time interval between symptom manifestation and last dose of radiation. Acute radiation proctitis refers to symptoms that occur within the first 3 months after radiation. Symptoms may include lower abdominal discomfort, diarrhoea, tenesmus, mucous discharge, urgency and minor rectal bleeding. Persistence of symptoms beyond 3 months after completion of radiotherapy or symptoms that begin 3 months after the initiation of radiation are defined as chronic radiation proctitis [1]. Haemorrhagic radiation proctitis (HRP) is defined as clinically significant rectal bleeding associated with characteristic mucosal changes such as telangiectasia, oedema, friability and/or ulceration [2]. Superficial vascular lesions develop due to ischemia from progressive intimal fibrosis with obliterative endarteritis [3]. The most severe forms of rectal bleeding often require repeated hospital admissions and blood transfusions. Argon plasma coagulation (APC) has been 